\title{
Análisis de desempeño a nivel de simulación de un sistema de comunicaciones Li-Fi para la transmisión de datos a alta velocidad ${ }^{1}$
}

\section{Simulation-level performance analysis of a Li-Fi communications system for high-speed data transmission}

\author{
E.A. Arteaga, G. H. Morán, y G.A. Gomez \\ Recibido: diciembre 5 de 2019 - Aceptado: julio 2 de 2020
}

\begin{abstract}
Resumen - Los altos requerimientos inalámbricos en acceso, capacidad y seguridad que demanda la sociedad actual junto con la gran congestión del espectro radioeléctrico ha llevado a buscar nuevas soluciones tecnologías como es la Fidelidad de la $\mathrm{Luz}(\mathrm{Li}$ Fi). Este artículo presenta el diseño e implementación de un sistema de comunicaciones Li-Fi dentro de un ambiente indoor utilizado la técnica de modulación OFDM óptica con recorte asimétrico (ACO-OFDM) a través del software MATLAB. EI desempeño del sistema se analiza en términos de la tasa de error de bit (BER) y la relación energía de bit a potencia de ruido (Eb/No) teniendo en cuenta la posición del receptor, orden de modulación QAM y velocidad de transmisión. Los resultados muestran que el desempeño del sistema depende directamente de la posición del receptor, además para altas tasas de transmisión el efecto multitrayectoria degrada el desempeño del sistema.

Palabras clave- ACO-OFDM, BER, Li-Fi, multitrayecto, simetría hermítica, VLC.
\end{abstract}

Abstract - The high wireless requirements in access, capacity and security demanded by today's society together with the great congestion of the radio spectrum has led to looking for new technological solutions such as Light Fidelity (Li-Fi). This paper

\footnotetext{
1 Producto derivado del proyecto de investigación "Análisis del desempeño a nivel de simulación de una red Li-Fi para la transmisión de datos", apoyado por la Universidad del Cauca a través del Grupo de investigación de Nuevas Tecnologías en Telecomunicaciones (GNTT) del Departamento de Telecomunicaciones.

E. A. Arteaga, Universidad del Cauca, Popayán, Colombia, email: estebanben@unicauca.edu.co

G. H. Moran, Universidad del Cauca, Popayán, Colombia, email: germanmoran@unicauca.edu.co

G. A. Gómez, Universidad del Cauca, Popayán, Colombia, email: gtgomez@unicauca.edu.co.

Como citar este artículo: Arteaga, E. A., Moran, G. H. y Gómez G. A. Análisis de desempeño a nivel de simulación de un sistema de comunicaciones Li-Fi para la transmisión de datos a alta velocidad, Entre Ciencia e Ingeniería, vol. 14, no. 27, pp. 73-81, enero-junio 2020. DOI: https://doi.org/10.31908/19098367.1786.
}

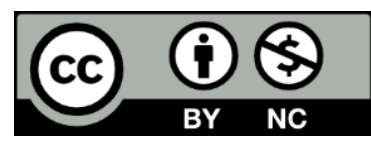

Attribution-NonCommercial 4.0 Intenational (CC By-NC 4.0) shows the design and implementation of a $\mathrm{Li}$-Fi communications system within an indoor environment used the optical OFDM modulation technique with asymmetric clipping (ACO-OFDM) through MATLAB software. System performance is analyzed in terms of bit error rate (BER) and bit to noise power ratio (Eb/No) taking into account receiver position, QAM modulation order and transmission rate. The results show that the performance of the system depends directly on the position of the receiver, in addition to high transmission rates the multipath degrades the performance of the system.

Keywords- ACO-OFDM, BER, Li-Fi, multipath, hermitian symmetry, VLC.

\section{INTRODUCCIÓN}

- $N$ los últimos años, las telecomunicaciones han alcanzado un gran desarrollo permitiendo la globalización del conocimiento e incidiendo sobre varios sectores tales como: el comercio, la salud, la educación y todos aquellos aspectos que requieren de algún modo el intercambio de información [1]. Las redes inalámbricas han desempeñado un papel importante en las comunicaciones modernas ya que gracias a sus características de movilidad, flexibilidad y conectividad facilitan la comunicación de forma ubicua [2].

Actualmente, la transmisión de información en las redes inalámbricas se realiza a través de tecnologías que operan en la banda de radiofrecuencia como: Wi-Fi, Bluetooth, NFC, redes celulares, redes satelitales, entre otras [3], cada una de estas tecnologías presenta sus propias características de desempeño y se utilizan dependiendo del tipo de aplicación. Sin embargo, con el auge del Internet de las Cosas (IoT, Internet of Things) y la industria 4.0, cada día aumenta el número de aplicaciones y dispositivos que se conectan a la red, por lo que los requisitos de acceso, capacidad y seguridad aumentan exponencialmente [4], [5]. Los grandes volúmenes de información requieren nuevas tecnologías que permitan soportar servicios en banda ancha, y ajustarse al tráfico existente.

En solución a las anteriores problemáticas y a la gran congestión del espectro radioeléctrico, investigadores han desarrollado una nueva tecnología conocida como Fidelidad de la Luz (Li-Fi, Light Fidelity) que consiste en la transmisión 
de datos mediante el uso del espectro visible. Los grandes benéficos de Li-Fi dentro de ambientes indoor han despertado el interés de muchas empresas y universidades lo que ha llevado a ser considerada como una tecnología de amplio potencial para las futuras comunicaciones [6], [7].

Li-Fi al igual que cualquier sistema de comunicaciones está compuesto por un transmisor, canal y receptor. El transmisor se encarga de convertir la señal eléctrica a óptica, se utilizan diodos LED como fuentes emisoras debido a su bajo costo y alta funcionalidad. La técnica de modulación que utilizan la mayoría de los sistemas de comunicación óptica inalámbrica (OWC, Optical Wireless Communication) es la modulación por intensidad y detección directa (IM/DD, Intensity Modulation and Direct Detection) en donde la forma de onda se modulada mediante la potencia óptica instantánea y se envía por el canal, por su parte el receptor capta las variaciones de intensidad y convierte la potencia óptica instantánea a corriente eléctrica para su posterior procesamiento.

Li-Fi es una tecnología de corto alcance, su desempeño se encuentra limitado por varios factores como el tipo de sistema, dimensiones del cuarto, posición y orientación del transmisor y receptor, coeficiente de reflexión de las paredes, entre otros. Los tipos de sistema Li-Fi se clasifican de acuerdo a la forma de propagación de la señal como: Línea de Vista (LOS, Line of Sight) y Visión Obstruida (NLOS, No-Line of Sight), los sistemas LOS alcanzan altas velocidades de transmisión, sin embargo se encuentran limitados porque debe existir visibilidad directa entre transmisor y receptor, en caso de que exista alguna obstrucción se produce un fallo total de la comunicación, caso contrario a los sistemas NLOS, donde los haces de luz se propagan por múltiples caminos lo que hace los sistemas sean robustos, pero se encuentran sujetos a otros fenómenos como las pérdidas de propagación y la interferencia entre símbolos (ISI, Inter Symbol Interference) causada por la superposición de los haces de luz en recepción. Por este motivo una solución ampliamente estudiada en la literatura para combatir los efectos multitrayectoria es el uso de la modulación multiportadora (OFDM), pero es necesario realizar algunas modificaciones al sistema clásico utilizado en radiofrecuencia para adaptarlo a las comunicaciones OWC [8]. Los sistemas ópticos OFDM (O-OFDM) se caracterizan porque la señal transmitida es real y positiva lo que permite modular la intensidad del LED.

Este artículo está organizado de la siguiente forma: en la sección II se presentan los trabajos relacionados; en la sección III se encuentran el modelo del sistema, donde se describen cada uno de los bloques que lo conforman; en la sección IV se presenta el análisis de resultados de la simulación y finalmente la sección V se presenta las conclusiones del trabajo.

\section{TRABAJOS RELACIONADOS}

Las comunicaciones por luz visible (VLC, Visible Light Communications) han sido objeto de investigación en los últimos años. Se han planteado una serie de investigaciones relacionadas con Li-Fi que van desde el estudio físico como: técnicas de modulación, distribución de potencia, modelado y análisis de dispositivos terminales: LEDs y fotodetectores, etc., hasta el diseño de arquitecturas y topologías de red basadas en esta tecnología, por ejemplo, en [8] se analiza el desempeño para las modulaciones ópticas DCO-OFDM y ACO-OFDM en términos de la relación potencia pico a potencia promedio (PAPR, Peak-to-Average Power Ratio) versus la tasa de error de bit sobre un canal AWGN, también se analiza el efecto que causa la no-linealidad del LED sobre la señal. Otros documentos como en [9], [10] analizan el desempeño del sistema utilizando técnicas de modulación hibridas que resultan de la combinación de la técnica ACOOFDM y la técnica multitono discreta modulada por amplitud de pulso (PAM-DMT, Pulse-Amplitude-Modulated Discrete Multitone Modulation). Para entender la tecnología es importante analizar cada uno de los efectos que pueden afectar su desempeño, por esta razón en [11] se analiza el desempeño del sistema en términos de BER para diferentes ángulos de visión (FOV, Field of View) del fotodetector.

\section{MODELO DEL SISTEMA}

El sistema por modelar es un sistema de única entradaúnica salida (SISO, Single Input-Single Output) dentro de una habitación vacía de dimensiones $(\mathrm{x}, \mathrm{y}, \mathrm{z})$. En esta sección se describe la modulación ACO-OFDM, también se presenta el modelado del canal óptico inalámbrico. En la Fig. 1, se muestra el escenario sobre el cual se realiza la simulación.

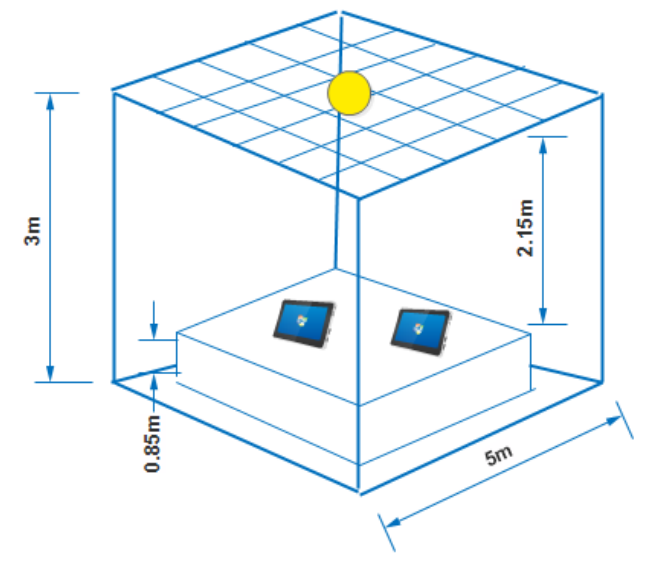

Fig. 1. Escenario de simulación.

\section{A. Modelo de canal OWC}

Para caracterizar el canal es necesario establecer el escenario de simulación, En la Tabla I se resumen las condiciones iniciales del sistema.

TABLA I.

PARÁMETROS DE SIMULACIÓN

\begin{tabular}{|l|c|c|}
\hline \multicolumn{1}{|c|}{ Parámetro } & Valor & Unidad \\
\hline Habitación & $\mathrm{m}$ \\
\hline Ancho & 5 & $\mathrm{~m}$ \\
\hline Alto & 5 & $\mathrm{~m}$ \\
\hline Altura del receptor & 3 & $\mathrm{~m}$ \\
\hline Coeficiente reflexión paredes & 0.85 & {$[\mathrm{adm}]$} \\
\hline \multicolumn{3}{|c|}{ Transmisor LED NICHIA [12]. } \\
\hline Angulo de media potencia & 60 & grados \\
\hline Potencia del LED & 236 & $\mathrm{~mW}$ \\
\hline \multicolumn{3}{|c|}{ Fotodetector BPW-34 [13]. } \\
\hline FOV & 60 & $\mathrm{grados}$ \\
\hline Área del fotodetector & 7.02 & $\mathrm{~mm}{ }^{\wedge} 2$ \\
\hline Responsividad & 0.62 & $\mathrm{~A} / \mathrm{W}$ \\
\hline
\end{tabular}


El canal OWC dentro de un ambiente indoor puede ser modelado como un sistema lineal en banda base e invariante en el tiempo [14]. Para el estudio del sistema Li-Fi se tiene en cuenta la fuente transmisora LED, el canal óptico y el fotodetector.

\section{1) $L E D$}

El modelo de distribución de potencia del LED tiene un patrón de radiación lambertiano, que se describe mediante la ecuación (1) [14], [15].

$$
R_{0}(\varphi)=\left\{\begin{array}{cc}
\frac{\left(m_{1}+1\right) \cos ^{m}(\varphi)}{2 \pi} & \frac{-\pi}{2} \leq \varphi \leq \frac{\pi}{2} \\
0 & \varphi \geq \frac{\pi}{2}
\end{array}\right\}
$$

Donde $m$ es el orden de emisión lambertiano que indica la directividad del haz de luz y $\varphi$ es el ángulo de radiación del LED. El orden de radiación lambertiano está relacionado con el semiángulo de media potencia mediante la ecuación (2).

$$
m=\frac{\ln _{2}}{\ln \left(\cos \left(\varphi_{1 / 2}\right)\right)}
$$

La potencia transmitida por el LED se expresa mediante la ecuación (3).

$$
P_{T X}=P_{L E D} * R_{0}(\varphi)
$$

\section{2) Fotodetector}

El fotodetector se modela como un área efectiva $A_{\text {eff }}$ que recolecta la radiación incidente mediante un ángulo $\Psi$ más pequeño que el FOV. El área efectiva del fotodetector se expresa mediante la ecuación (4).

$$
A_{e f f}=\left\{\begin{array}{cr}
A_{R} \cos (\Psi), & 0<\Psi \leq \frac{\pi}{2} \\
0 & \Psi \geq \frac{\pi}{2}
\end{array}\right.
$$

Donde $\Psi$ es el ángulo de incidencia y $A_{R}$ el área total del fotodetector. Para aumentar el $A_{\text {eff }}$ y por lo tanto la cantidad de flujo luminoso que incide sobre la superficie del fotodetector es necesario usar un concentrador sin imágenes. La ganancia óptica del concentrador ideal sin imágenes con índice de refracción $n$ se expresa mediante la ecuación (5).

$G(\psi)=\left\{\begin{array}{cc}\frac{n^{2}}{\sin ^{2}\left(\psi_{c}\right)} & 0<\psi \leq \psi_{c} \\ 0 & \psi \geq \psi_{c}\end{array}\right.$

Donde $\psi_{c}$ es el FOV y se debe satisfacer que $\psi_{c} \leq \frac{\pi}{2}$

\section{3) Canal Óptico Inalámbrico.}

En el canal OWC los haces de luz se propagan de 2 maneras diferentes: Línea de Vista o multitrayectoria. En la
Fig. 2, se muestra el escenario de simulación, donde se observa la geometría del sistema cuando la señal se propaga por estos 2 modos.

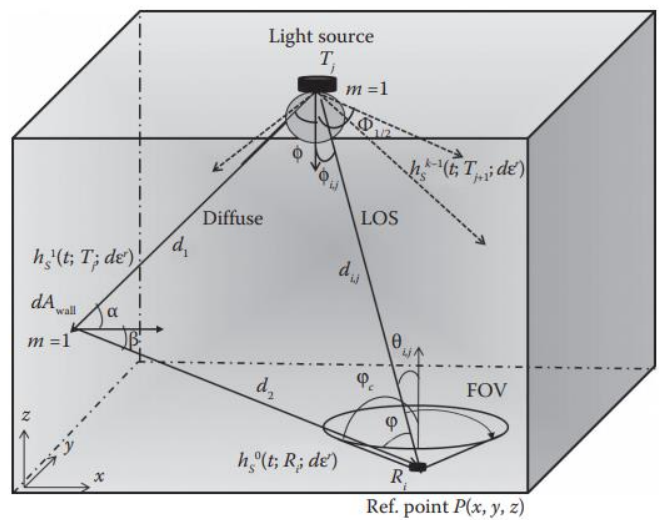

Fig. 2. Geometría transmisor, receptor y reflexiones [15].

La ganancia DC de canal cuando existe LOS se representa mediante la ecuación (6).

$$
H_{l o s}(0)=\left\{\begin{array}{cr}
\frac{A_{R}^{*} R_{0}(\varphi) * T_{s}(\psi)^{*} G_{s}(\psi) * \operatorname{Cos}(\Psi)}{d^{2}}, & 0<\psi \leq \psi c \\
0 \quad, & \psi \geq \psi c
\end{array}\right.
$$

Donde $A_{R}$ es el área del fotodetector, $d$ es la distancia entre el trasmisor y el receptor, $R_{0}(\varphi)$ la intensidad radiada por el LED, $\psi$ es el ángulo de incidencia, $T_{S}(\psi)$ y $G_{S}(\psi)$ representan la ganancia del filtro pasa bajos y la del concentrador sin imágenes [16]. De este modo la potencia recibida se expresa mediante la ecuación (7).

$$
P_{R x, L O S}=P_{T x} * H_{L O S}^{1}(0)
$$

Donde $H_{l o s}^{i}(0)$ representa la i-esima ganancia DC del canal. Por otro lado, los efectos multitrayectoria son más difíciles de predecir, ya que dependen de varios factores como: las dimensiones de la habitación, posición y orientación del transmisor y receptor, coeficiente de reflexión de las paredes, entre otros. La ganancia DC de cada uno de los caminos reflejados se expresa mediante la ecuación (8).

$H_{r e f}(0)=\left\{\begin{array}{cc}\frac{A_{r} * R_{0}(\varphi) * \rho * d A \text { wall } * \cos (\alpha) * \cos (\beta) * T_{S}(\psi) * G_{S}(\psi) * \cos (\psi)}{d_{1}^{2} * d_{2}^{2}}, & 0 \leq \Psi \leq \Psi_{c} \\ 0 & , \Psi \geq \Psi_{c}\end{array}\right.$

Donde $\alpha$ es el ángulo de incidencia con el que el rayo se refleja en la pared, $\beta$ representa el ángulo de irradiación desde el área reflectante de la pared hacia el plano receptor, $d_{1}$ es la distancia entre el transmisor y la pared, $d_{2}$ es la distancia entre la pared y un punto en la superficie receptora y $d$ Awall es el tamaño del área reflectante. La potencia recibida teniendo en cuenta Línea de Vista y los efectos de multitrayectoria se expresa mediante la ecuación (9). 


$$
P_{R x}=\left(H_{L O S}(0)+\sum_{r e f} H_{r e f}(0)\right) * P_{T x}
$$

\section{B. Técnica de Modulación Óptica ACO-OFDM}

La modulación óptica ACO-OFDM se caracteriza porque las subportadoras impares llevan los datos, mientras que las subportadoras pares forman una señal de polarización que garantiza que la señal OFDM no tenga componentes negativas. En la Fig. 3, se muestra el diagrama de bloques para un sistema que emplea modulación ACO-OFDM, donde la señal $\mathbf{X}$ a la entrada de la IFFT se organiza teniendo en cuenta la estructura de la ecuación (10) y (11) [17], [18].

$$
\begin{aligned}
& S_{\text {ACO }}=\left\{0, X_{0}, 0, X_{1}, \ldots, X_{N-1}, 0, \overline{X_{N-1}}, 0, . ., \overline{X_{1}}, 0, \overline{X_{0}}\right\} \\
& S_{\text {ACO }}=\left\{0, S_{1}, 0, S_{3}, . . S_{M / 2-1}, 0, \overline{S_{M / 2-1}}, 0, . ., \overline{S_{3}}, 0, \overline{S_{1}}, 0\right\}
\end{aligned}
$$

Donde de (10), $X_{0}, X_{1}, \ldots, X_{N-1}$ representan cada uno de los símbolos que se obtiene después de la modulación M-Aria y de (11), $S_{1}, S_{3}, ., S_{M / 2-1}$ son cada una de las subportadoras impares sobre las cuales se trasportará la información. Cada elemento de $S_{\mathrm{ACO}}$ cumple con la propiedad de simetría hermítica para garantizar que la salida de la IFFT sea una señal real.

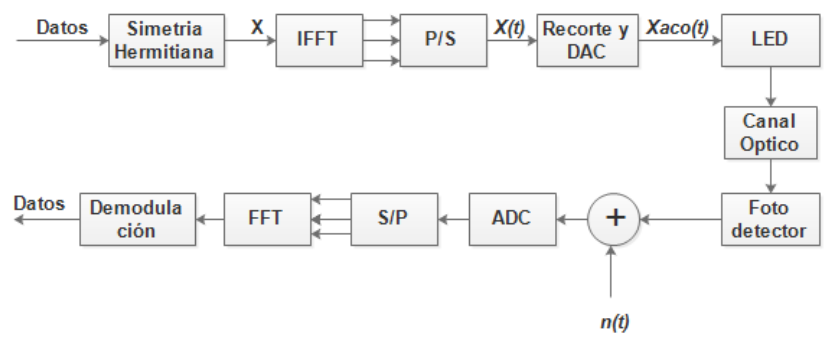

Fig. 3. Sistema Li-Fi con modulación ACO-OFDM [18].

Posteriormente se realiza la conversión de paralelo/serie de los datos y se realiza un recorte a cero para garantizar que la señal de salida $x(t)$ sea completamente positiva. El recorte no genera pérdida de datos porque los datos se encuentran contenidos dentro de las subportadoras impares, sin embargo, la intermodulación causada por el recorte afectará a las componentes pares. Finalmente se obtiene una señal real y positiva, $x_{a c o}(t)$ a la cual se le realiza la conversión DAC para modular la intensidad del LED y enviarla por el canal óptico.

En recepción se realiza procedimiento inverso, el fotodetector capta las variaciones de intensidad del LED y realiza la conversión óptica/eléctrica, el ruido se añade en el dominio eléctrico y se modela como AWGN [14], después se filtra la señal y se realiza la conversión ADC. La señal discreta en tiempo pasa por el bloque FFT para convertirla en una señal discreta en frecuencia, a la cual se le retiran las portadoras impares y se las pasa por un bloque demodulador para obtener la señal original. En ACO-OFDM solo una cuarta parte de las subportadoras se utilizan para trasportar información por lo que espectralmente es una técnica ineficiente.

\section{Diseño de la Herramienta de Simulación Li-Fi}

Para facilitar el análisis del sistema de comunicaciones LiFi se desarrolló una herramienta grafica denominada LiFindor mediante la GUI de MATLAB que permite analizar el comportamiento del sistema dentro de un escenario indoor variando diferentes parámetros como: número de subportadoras, orden de modulación, tipo de LED, valor de $\mathrm{Eb} / \mathrm{No}$, dimensiones del escenario, entre otros. La herramienta proporciona un análisis secuencial de cada uno los procesos que sufre la señal a medida que avanza por el sistema con el fin de obtener la tasa de error de bit para la modulación ACOOFDM. La herramienta también proporciona un análisis de cobertura teniendo en cuenta la propagación de la señal cuando existe Línea de Vista y multitrayectoria. En la Fig. 4, se muestra la interfaz gráfica de la herramienta.

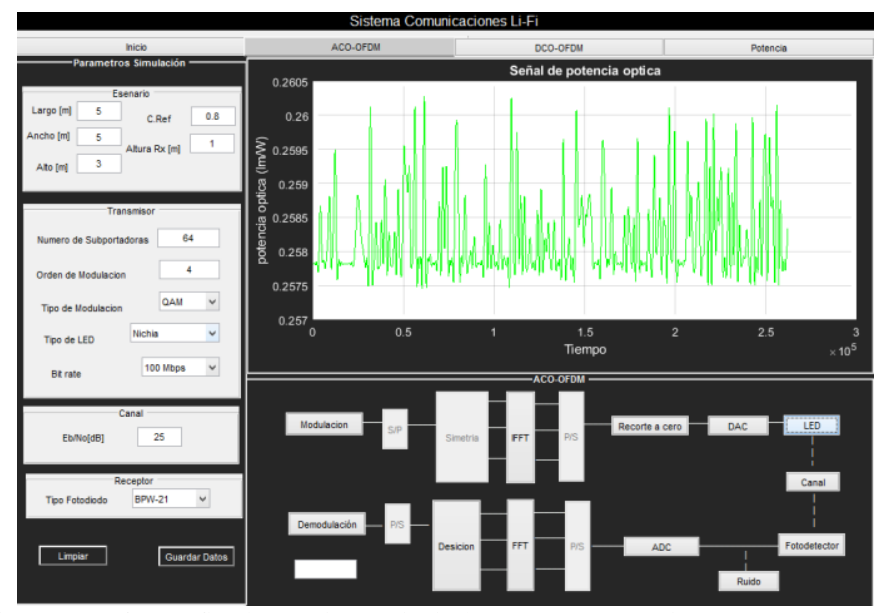

Fig. 4. Interfaz gráfica herramienta Li-Findoor - Fuente Propia.

\section{SiMUlaCIONES Y RESUltadOS}

El análisis de desempeño del sistema de comunicaciones Li-Fi se centra en base a la cobertura, la tasa de error de bit y la eficiencia espectral, de acuerdo con la posición del receptor y el orden de modulación M-QAM empleado.

\section{A. Cobertura}

Para el análisis de cobertura se tienen en cuenta 3 variables: distribución de potencia, máxima dispersión del retardo y perfil potencia-retardo.

\section{1) Ditribución de Potencia}

En la Fig. 5, se muestra la distribución de potencia del sistema cuando existe Línea de Vista, donde se observa claramente que la mayor concentración de energía se encuentra en el centro de la habitación a $-6 \mathrm{dBm}$ y a medida que el receptor se aleja del centro de la habitación la potencia cae hasta $-16 \mathrm{dBm}$. Cabe resaltar que para todos los escenarios de simulación el LED se encuentra ubicado en el centro de la habitación.

En la Fig. 6, se muestra la distribución de potencia cuando únicamente hay presencia de multitrayectoria, es decir cada uno de los rayos de luz llegan al receptor de manera diferente al rayo principal. En este caso se observa que la mayor contribución de potencia se encuentra en el borde de las paredes a $-20 \mathrm{dBm}$, mientras que en centro de la habitación el nivel de potencia cae hasta $-29 \mathrm{dBm}$, esto se debe a que la 
señal en el borde de las paredes experimenta menos atenuación con respecto al centro de la habitación, de (8) se sabe que existe una relación inversa entre atenuación y distancia.

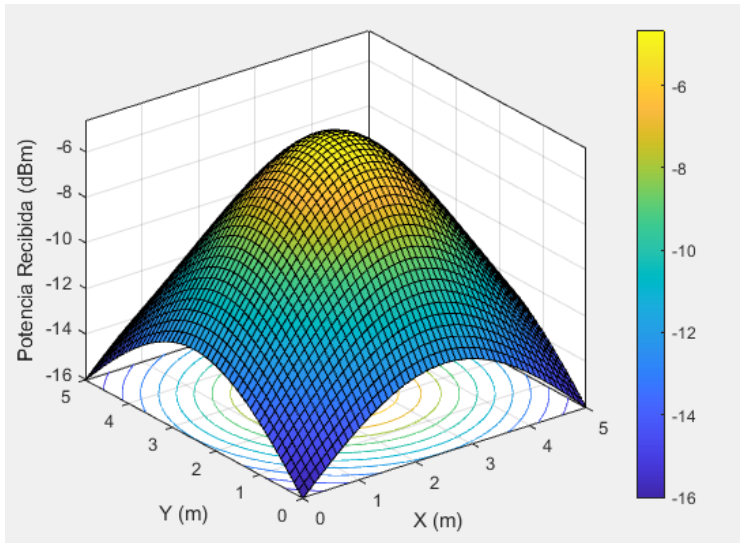

Fig. 5. Distribución de potencia Línea de Vista.

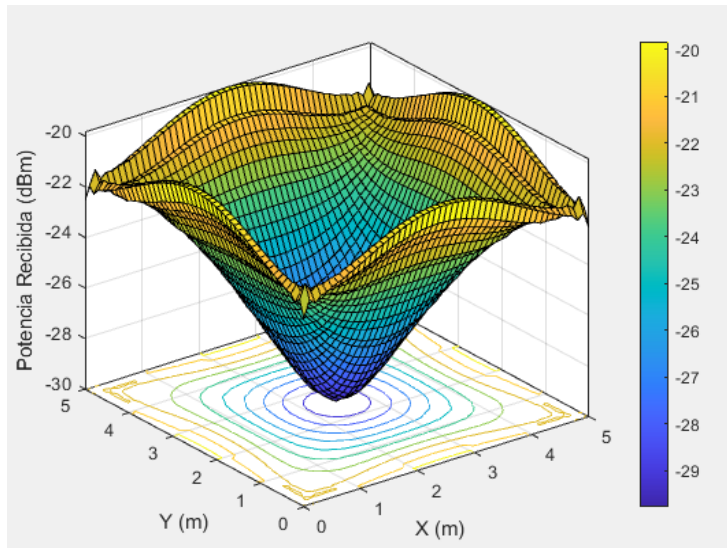

Fig. 6. Distribución de potencia multitrayectoria.

Finalmente, en la Fig. 7, se muestra la distribución de potencia total, teniendo en cuenta los rayos LOS y NLOS donde se observa que no hay mucha diferencia con respecto a la Fig. 5, excepto en los bordes de la habitación donde hay un aumento de $1 \mathrm{dBm}$, que es proporcionado por la contribución de los rayos multitrayectoria.

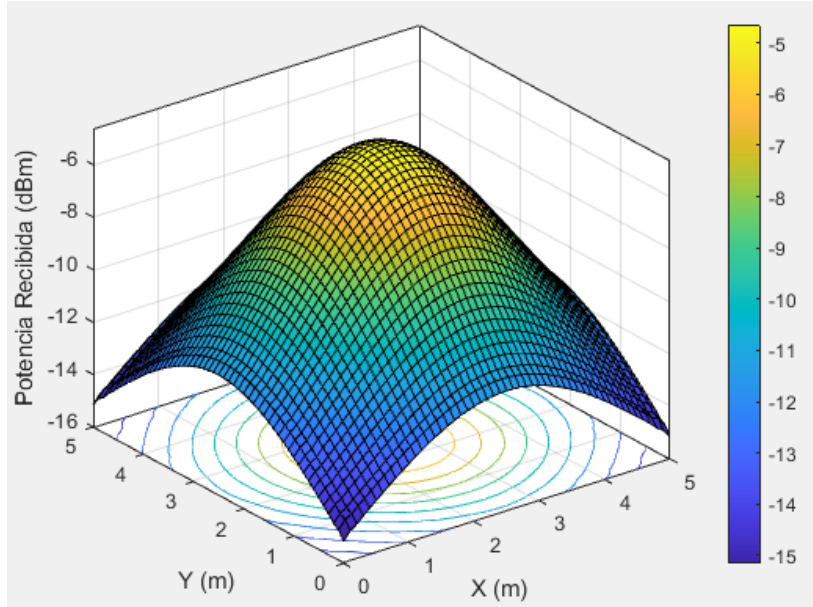

Fig. 7. Distribución de potencia total.

\section{2) Máximo Retardo}

El máximo retardo cuadrático medio (DRMS, Root Mean Delay Spreed) es un parámetro que permite cuantificar las propiedades dispersivas en tiempo de los canales multitrayecto y se define mediante la ecuación (12) [14].

$$
D_{R M S}=\left[\frac{\int(t-\mu)^{2} h^{2}(t) d t}{\int h^{2}(t) d t}\right]^{1 / 2}
$$

Donde $h(t)$ es la respuesta al impulso del sistema y $\mu$ es la media del retardo que se define mediante a la ecuación (13).

$$
\mu=\frac{\int t h^{2}(t) d t}{\int h^{2}(t) d t}
$$

En la Fig. 8, se muestra el valor del $D_{R M S}$ para cada punto del plano de recepción, donde se observa que en el centro de la habitación existe el menor valor de $0.05 n$, esto es evidente debido a que la componente de rayo directo es la que predomina sobre los rayos multitrayecto, en cambio en las esquinas de la habitación se observa el mayor valor $D_{R M S}$ es aproximadamente de $0.35 \mathrm{~ns}$, esto es debido que las componentes multitrayecto empiezan a ser relevantes e imponerse sobre la componente de rayo directo.

A través de este parámetro se determina la máxima velocidad alcanzable por medio de la ecuación (14), la cual se utiliza para establecer un límite de desempeño en el análisis de la velocidad por medio de las curvas Eb/No [15].

$$
R_{b} \leq \frac{1}{10 D_{\text {RMS }}}
$$

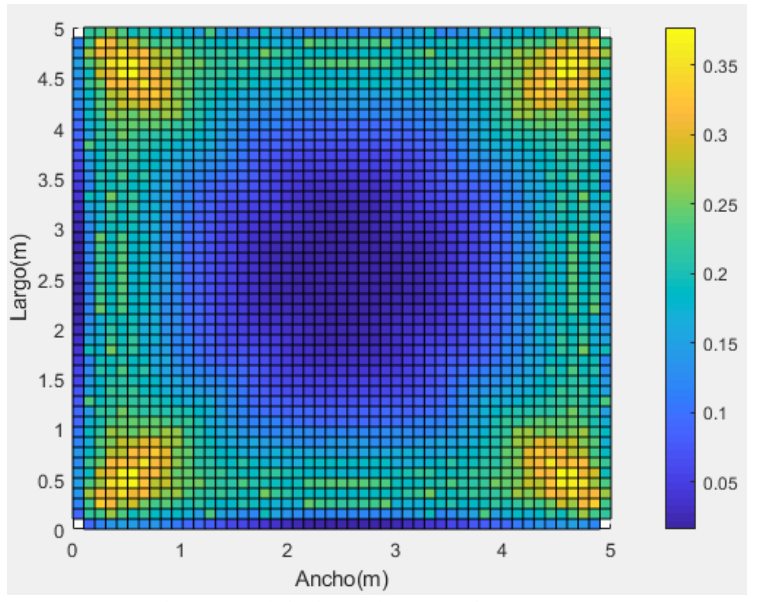

Fig. 8. Drms para cada punto del plano de recepción.

\section{3) Perfil de Potencia-Retardo}

El perfil de potencia-retardo (PDP, Power Delay Profile) permite analizar el comportamiento de canales multitrayecto, en términos de la potencia recibida y el tiempo de llegada de cada rayo de luz. Se seleccionan 3 posiciones diferentes para el receptor y se analiza el efecto de la multitrayectoria para cada escenario. En la Fig. 9, se muestra el perfil de potenciaretardo para un receptor ubicado justamente debajo del LED en el punto P1(2.5,2.5,0.85), donde se observa que la componente LOS es la más significativa y llega en un tiempo 
de 7.125ns, mientras que las componentes multitrayectoria no son significativas por lo que se consideran despreciables.

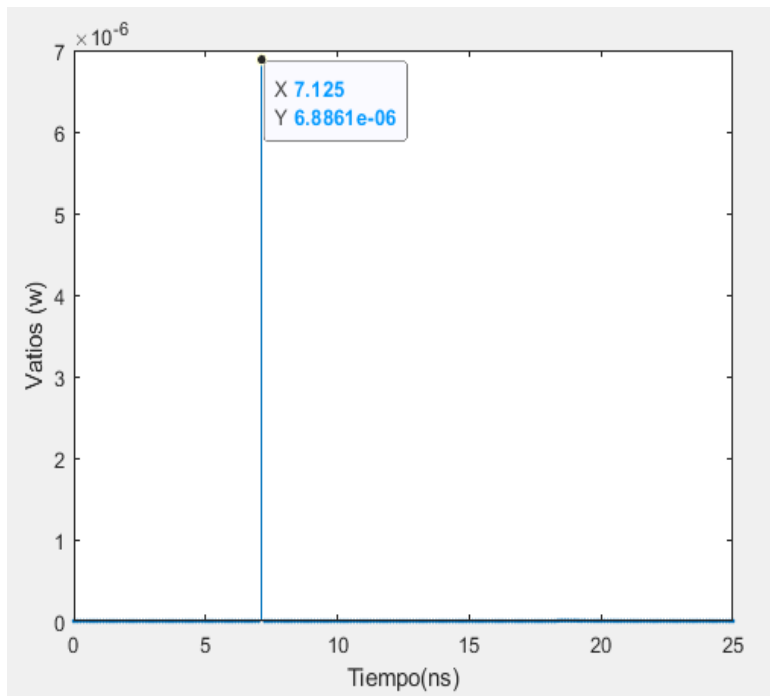

Fig. 9. Perfil de potencia-retardo punto P1 $(2.5,2.5,0.85)$.

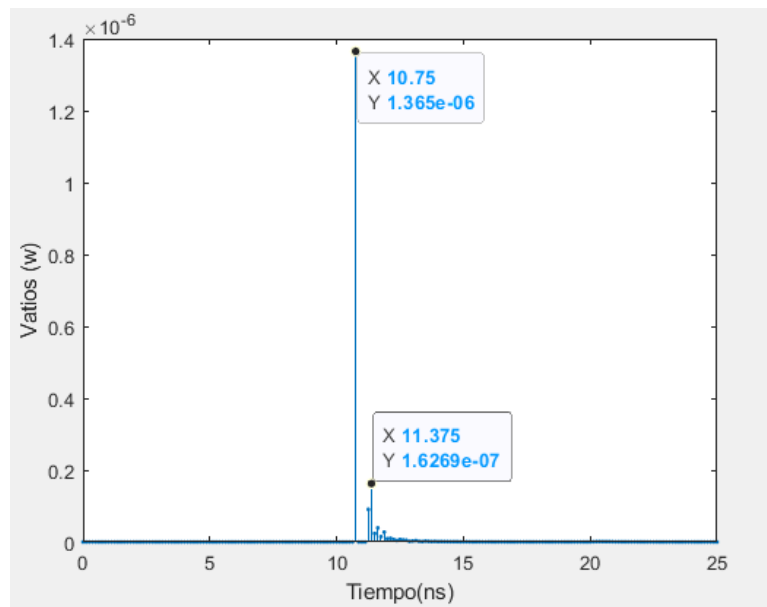

Fig. 10. Perfil de potencia-retardo punto P2 $(2.5,4.9,0.85)$.

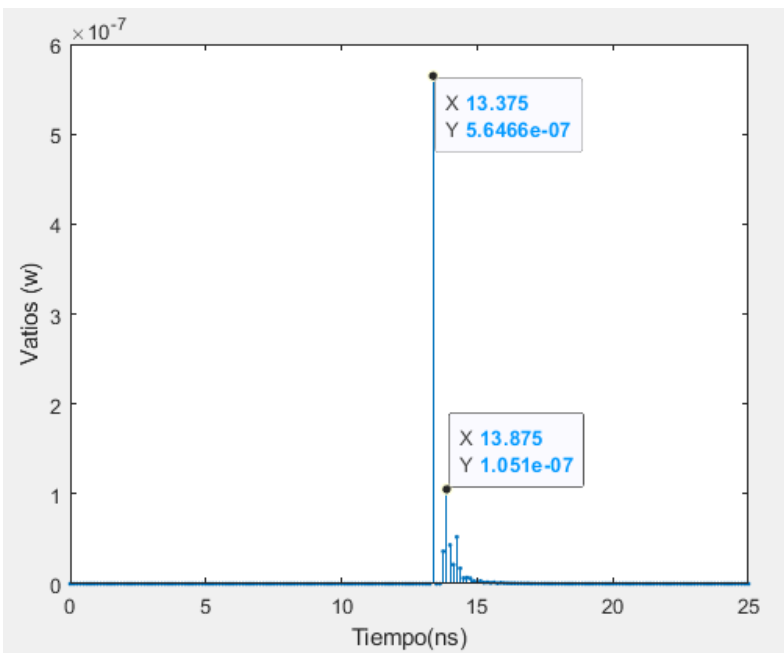

Fig. 11. Perfil de potencia-retardo punto P3 (4.9, 4.9, 0.85).

Para el caso del receptor ubicado en el punto P2(2.5,4.9, 0.85) como se muestra en la Fig. 10, se observa que la componente LOS sigue siendo la más significativa y llega al receptor en un tiempo de 10.75ns, sin embargo, las componentes de la multitrayectoria ya no son despreciables, porque llegan en un tiempo cercano al rayo principal y aunque aportan bajos niveles de potencia, la sumatoria de todos los rayos contribuye a la señal final.

Para este caso la multitrayectoria contribuye a mejorar el desempeño del sistema en términos de potencia recibida.

Ahora, en la Fig. 11, se muestra el perfil de potencia-retardo para un receptor ubicado en la esquina de la habitación, es decir en el punto P3(4.9,4.9,0.85), esta es la zona más crítica y se observa que la componente LOS sigue siendo la más significativa, pero las componentes multitrayectoria ya no son despreciables porque aportan un nivel considerable de potencia a la señal.

\section{4) BER vs Eb/No}

El análisis de desempeño de un sistema de comunicación digital generalmente se realiza mediante las curvas de BER vs $\mathrm{Eb} / \mathrm{No}$, para el sistema de comunicaciones propuesto se analiza el desempeño variando el orden de modulación MQAM para diferentes posiciones del receptor a diferentes velocidades y de esta manera determinar la máxima tasa de datos alcanzable. Se ha seleccionado 3 posiciones diferentes dentro de la habitación: centro, esquina y pared.

Como caso inicial se analiza el desempeño del sistema únicamente con la presencia de ruido AWGN sin considerar el efecto de multitrayectoria.

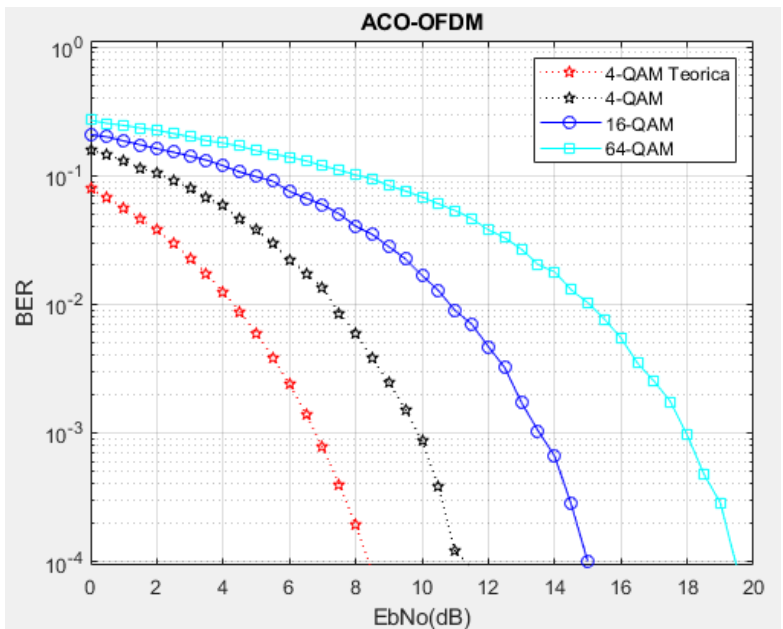

Fig. 12. Curva de desempeño canal AWGN.

En la Fig. 12, se puede observar que para el esquema de modulación 4-QAM de ACO-OFDM se requiere $3 \mathrm{~dB}$ más de potencia que para el esquema de modulación 4-QAM bipolar, esto se debe a que en ACO-OFDM se utiliza la mitad de la potencia eléctrica para transmitir solo en la mitad de las subportadoras, las subportadoras impares son las que transportan la información, mientras que las subportadoras pares se utiliza como condición de simetría hermítica para convertir la señal a unipolar, para todos los esquemas de modulación es igual, siempre se requiere $3 \mathrm{~dB}$ adicionales para poder transmitir con ACO-OFDM [19].

\section{5) Centro de la habitación.}

En la Fig. 13, se observa el comportamiento del sistema 
cuando el fotodetector se encuentra ubicado justamente debajo del LED y se transmite a una velocidad de $100 \mathrm{Mbps}$, la curva de desempeño es muy similar a la curva del canal AWGN, esto se debe a que las componentes multitrayectoria se consideran despreciables solo hay contribución del rayo directo.

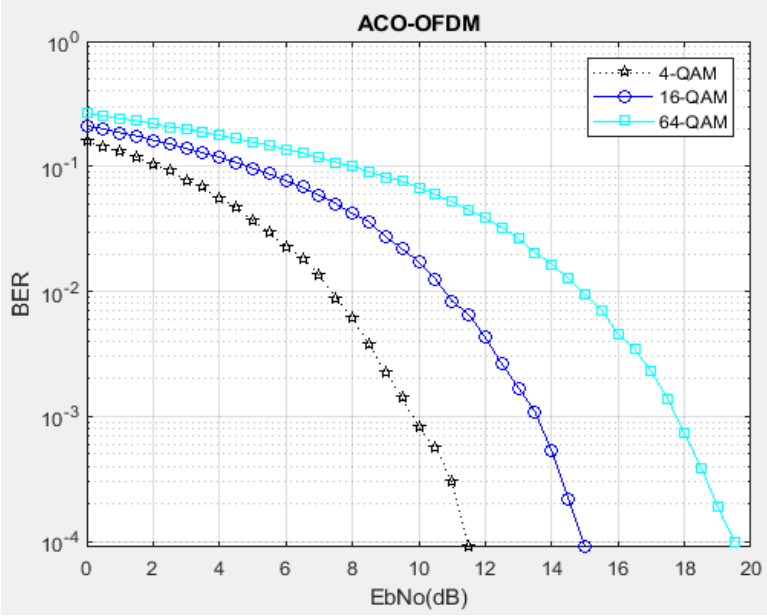

Fig. 13. Curva de desempeño para el receptor ubicado en el punto P1 (2.5, $2.5,0.85)$ a $100 \mathrm{Mbps}$.

En la Fig. 14, se observa el comportamiento del sistema LiFi en el centro de la habitación cuando se transmite a una velocidad de $2 \mathrm{Gbps}$. El comportamiento es similar a un canal AWGN, debido a que el efecto multitrayectoria es muy bajo, por lo tanto, la atenuación que sufren los rayos difusos no aporta niveles importantes a la señal final. Cabe aclarar que el estudio se realiza sin considerar limitaciones de ancho de banda.

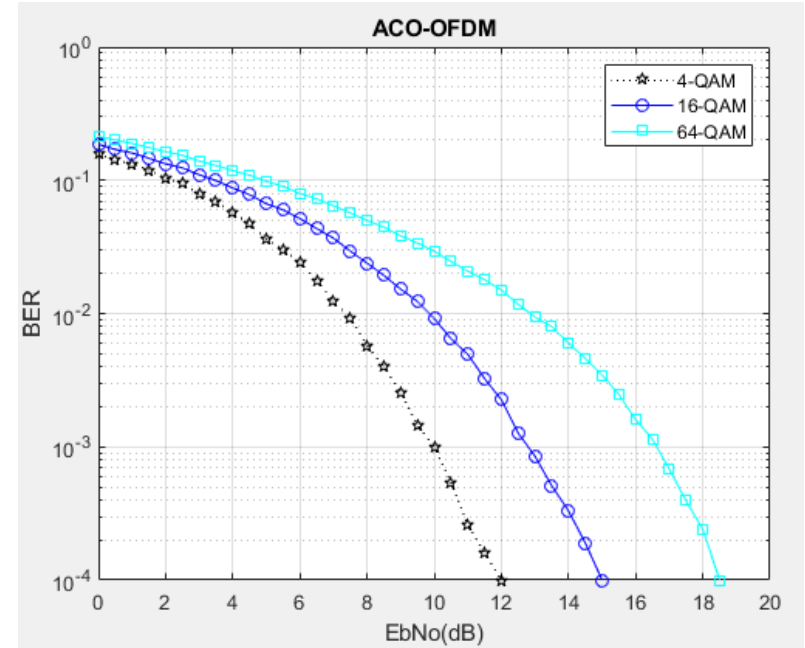

Fig. 14. Curva de desempeño para el receptor ubicado en el punto P1 (2.5, $2.5,0.85)$ a $2 \mathrm{Gbps}$.

\section{6) Pared de la habitación.}

En la Fig.15, se observa el comportamiento del sistema cuando el fotodetector se ubica en la pared de la habitación, para los esquemas de modulación 4-QAM y 16-QAM se requiere una $\mathrm{Eb} / \mathrm{No}$ adecuada, sin embargo, para 64- QAM, la multitrayectoria causa ISI que se ve reflejado en el desempeño del sistema ya que se requiere una Eb/No superior a $20 \mathrm{~dB}$ para alcanzar una BER específica.

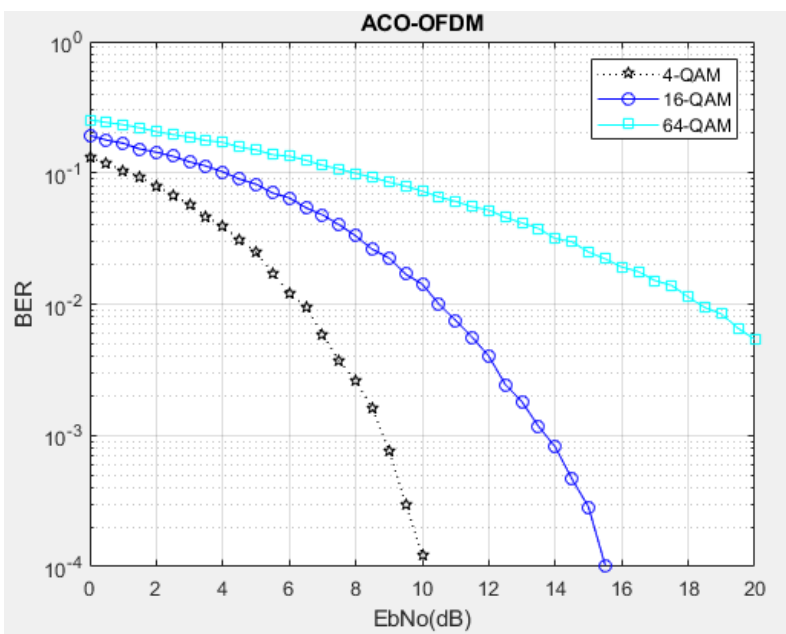

Fig. 15. Curva de desempeño para el receptor ubicado en el punto P2 (2.5, $4.9,0.85)$ a $100 \mathrm{Mbps}$.

El desempeño del sistema en la pared de la habitación a una velocidad de 2 Gbps se presenta en la Fig.16, donde se observa que los requerimientos de $\mathrm{Eb} / \mathrm{No}$ aumentan con respecto a la velocidad de 100Mbps, sin embargo, se mantiene el comportamiento para una modulación de 64-QAM porque el efecto multitrayectoria es considerable.

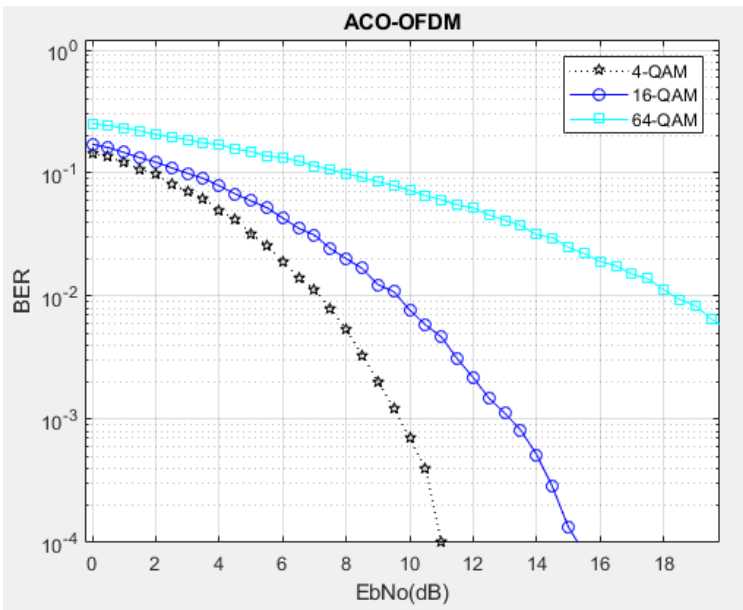

Fig. 16. Curva de desempeño para el receptor ubicado en el punto P1 (2.5, $4.9,0.85)$ a 2 Gbps.

\section{7) Esquina de la habitación.}

En la Fig. 17, se observa el comportamiento del sistema $\mathrm{Li}-\mathrm{Fi}$, cuando el receptor se ubica en la esquina de la habitación y se transmite a $100 \mathrm{Mbps}$, como se puede apreciar para bajos ordenes de modulación los requerimientos de $\mathrm{Eb} / \mathrm{No}$, no aumentan mucho con respecto al receptor ubicado en el centro de la habitación, sin embargo, cuando se utiliza un alto orden de modulación como 64-QAM se llega a un punto donde la BER es irreductible aun cuando se aumenta la $\mathrm{Eb} / \mathrm{No}$, esto es debido al efecto de la multitrayectoria, que causa una rotación en la constelación recibida y por lo tanto una mala estimación de los símbolos recibidos, porque la estimación de la señal ya no depende del símbolo recibido en ese instante, sino de símbolos anteriores. 


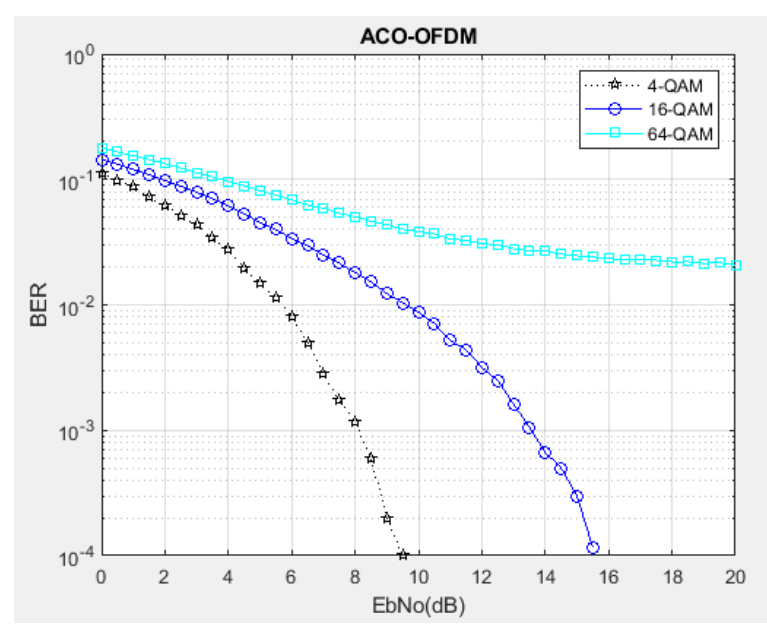

Fig. 17. Curva de desempeño para el receptor ubicado en el punto P3 (4.9, $4.9,0.85)$ a $100 \mathrm{Mbps}$.

Como se observa en la Fig. 18, al aumentar la velocidad a 2Gbps, los requerimientos de Eb/No aumentan en esquemas de modulación bajos. Para poder transmitir con 4-QAM o 16QAM, se requieren aproximadamente de $1 \mathrm{~dB}$ a $2 \mathrm{~dB}$ más que para transmitir a $100 \mathrm{Mbps}$, sin embargo, para un esquema de modulación alto como 64-QAM se mantiene el comportamiento de una BER irreductible. Ahora si se compara el desempeño del sistema a $2 \mathrm{Gbps}$ con respecto al centro de la habitación de la Fig. 13, se observa que no existen gran diferencia para los bajos esquemas de modulación, el problema se presenta cuando se utiliza 64-QAM. Cabe resaltar que en este estudio no se utiliza un prefijo cíclico (CP, Cyclic Prefix) para mitigar los efectos de la multitrayectoria, de igual manera no se hace uso de ningún tipo de ecualizador en recepción.

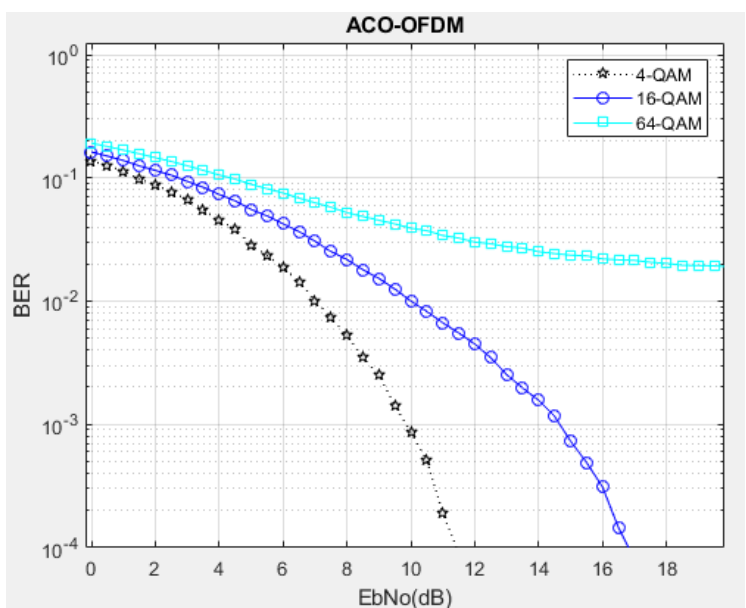

Fig. 18. Curva de desempeño para el receptor ubicado en el punto P3 (4.9, $4.9,0.85)$ a $2 \mathrm{Gbps}$.

\section{8) Eficiencia Espectral.}

La eficiencia espectral en un sistema de comunicaciones es la relación entre cantidad de bits por segundo que se puede transmitir en un determinado ancho de banda. Según [9] la eficiencia espectral en un sistema con modulación ACOOFDM viene dada mediante la ecuación (15).

$$
\eta_{A C O-O F D M}=\frac{\left(\frac{N / 4-1}{N+N_{g}}\right) B \log _{2} M}{B}
$$

Donde $B$ denota el ancho de banda, $N g$ denota el número de subportadoras de guarda utilizadas en el CP y $M$ es el orden de modulación, como en este estudio no se utiliza el CP y $N$ ? 1 , (15) puede ser expresada mediante la ecuación (16).

$$
\eta_{A C O-O F D M}=\frac{1}{4} \log _{2} M
$$

De (16) se observa que la eficiencia espectral del sistema ACO-OFDM depende únicamente del orden de modulación y solo utiliza la cuarta parte de las subportadoras para transmitir información.

\section{CONCLUSIONES}

La máxima tasa de datos alcanzable para un sistema Li-Fi depende de la posición del receptor y el orden de modulación M-QAM utilizado. En el centro de la habitación es posible alcanzar la máxima tasa teórica de $2 \mathrm{Gbps}$ utilizando todos los esquemas de modulación M-QAM, no obstante, a medida que el receptor se aleja del centro hacia la pared o esquina de la habitación, los requerimientos de Eb/No aumentan para mantener una BER específica, sin embargo, en la esquina de la habitación se presenta una BER irreductible para 64-QAM.

Aunque es posible alcanzar la máxima tasa de datos teórica, en este estudio no se considera el efecto del ancho de banda de modulación del LED, de igual manera no se utiliza ningún tipo de ecualizador en la recepción por lo que el efecto de la ISI es considerable en esquemas de modulaciones altos.

Li-Fi se considera una tecnología prometedora para el futuro de las telecomunicaciones mundiales, su amplia capacidad de despliegue, y su alta velocidad de transmisión de datos supera en gran medida a tecnologías inalámbricas de ambiente indoor como Wi-Fi, además de la gran ventaja de operar en la banda del espectro de luz visible que alivia la congestión del espectro radioeléctrico, sin embargo posee ciertas limitaciones que deben ser estudiadas ya que el desempeño del sistema depende de la visibilidad directa entre transmisor y receptor. Se espera que en un futuro cercano $\mathrm{Li}$ Fi y Wi-Fi pueda operar de manera conjunta.

\section{AGRADECIMIENTOS}

Los autores reconocen las contribuciones del departamento de Telecomunicaciones de la Universidad del Cauca por sus aportes y recomendaciones y al grupo de investigación de Nuevas Tecnologías en Telecomunicaciones (GNTT) de la Universidad del Cauca, por su colaboración y asesoría en el desarrollo del presente artículo, de igual manera a los ingenieros Mauricio Ramírez y Manuela Silva.

\section{REFERENCIAS}

[1] S. Douglas and T. Ely., "The Tecnologies of the information and communications necessary to the society of the information of XXI century," СИМВОЛ НАУКИ, vol. 3, no.3, pp. 173-176, 2016. 
[2] J. Gómez., "Servicios en Red,” 1ra ed, Ed. Editex, pp. 212-214, 2010.

[3] S. Dhawan, "Analogy of Promising Wireless Technologies on Different Frequencies: Bluetooth, WiFi, and WiMAX," in The 2nd International Conference on Wireless Broadband and Ultra Wideband Communications, Sidney, pp. 14-14, 27-30 Aug. 2007.

[4] S. Singh and N. Singh, "Internet of Things (IoT): Security challenges, business opportunities \& reference architecture for E-commerce," in 2015 International Conference on Green Computing and Internet of Things (ICGCIoT), Noida, pp. 1577-1581, 8-10 Oct. 2015.

[5] M. O. Gokalp, K. Kayabay, M. A. Akyol, P. E. Eren, and A. Kocyigit, "Big Data for Industry 4.0: A Conceptual Framework," in 2016 International Conference on Computational Science and Computational Intelligence (CSCI), Las Vegas, pp. 431-434, 15-17 Dec. 2016.

[6] M. Monisha and G. Sudheendra, "Lifi- Light Fidelity Technology," in International Conference on Current Trends in Computer, Electrical, Electronics and Communication (CTCEEC), Mysore, pp. 818-821, 8-9 Sept. 2017.

[7] V. Shah, S. Chaudhary and B. Jain, "Architecture based on Li-Fi to enhance the working environment," Int. J. Adv. Res. Comput. Commun. Eng. ISO, vol. 6, no. 2, pp. 226-229, Feb. 2017.

[8] R. Mesleh, H. Elgala, and H. Haas, "On the Performance of Different OFDM Based Optical Wireless Communication Systems," J. Opt. Commun. Netw., vol. 3, no. 8, pp. 620-628, Aug. 2011.

[9] S. D. Dissanayake and J. Armstrong, "Comparison of ACO-OFDM, DCO-OFDM and ADO-OFDM in IM/DD Systems," J. Light. Technol., vol. 31, no. 7, pp. 1063-1072, Apr. 2013.

[10] B. Ranjha and M. Kavehrad, "Hybrid asymmetrically clipped OFDMbased IM/DD optical wireless system," J. Opt. Commun. Netw., vol. 6, no. 4, pp. 387-396, May. 2014

[11] S. J. Lee and S. Y. Jung., "A SNR analysis of the visible light channel environment for visible light communication," in 2012 - 18th AsiaPacific Conference on Communications (APCC), " Jeju Island, pp. 709712, 15-17 Oct. 2012

[12] Nichia Corporation, "Technical Datasheet White LED NF2W757GRTV1 ", 2016.

[13] OSRAM Opto Semiconductors, "Technical Datasheet photodetectro BPW-34", 2018

[14] Z. Ghassemlooy, W. Popoola, and S. Rajbhandari, "Optical wireless communications: system and channel modelling with MATLAB," 2nd ed, Boca Raton, Ed. CRC Press Taylor and Francis Group, 2019.

[15] Z. Ghassemlooy, L. N. Alves, S. Zvanovec, and M. Khalighi, "Visible light communications: theory and applications," 1ra ed, Boca Raton, Ed. CRC Press Taylor and Francis Group, 2017.

[16] L. Zeng, D. O'Brien, H. Le-Minh, K. Lee, D. Jung, and Y. Oh, "Improvement of Date Rate by using Equalization in an Indoor Visible Light Communication System," in 2008 4th IEEE international Conference on Circuits and Systems for Communications, Shanghai, pp. 678-682, 26-28 May. 2008.

[17] X. Zhang, Y. Zhou, Y. Yu, P. Han, and X. Wang, "Comparison and Analysis of DCO-OFDM, ACO-OFDM and ADO-OFDM in IM/DD Systems," Applied Mechanics and Materials., vol. 701-702, pp. 10591062, Dec. 2014.

[18] S. C. Saju and A. J. George., "Comparison of ACO-OFDM and DCOOFDM in IM/DD Systems," Int. J. Eng. Res. Tecnol., vol. 4, no. 04, pp. 1315-1318, Apr. 2015.

[19] J. Armstrong and B. Schmidt, "Comparison of asymmetrically clipped optical OFDM and DC-biased optical OFDM in AWGN," IEEE Commun. Lett., vol. 12, no. 5, pp. 343-345, May. 2008.

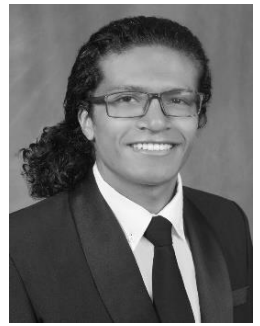

Esteban Alberto Arteaga Benavides: Nació en Pupiales, Nariño, Colombia, el 2 de septiembre de 1995. Ingeniero en Electrónica Telecomunicaciones de la Universidad del Cauca. Entre sus áreas de interés están, sistema de comunicaciones inalámbricos, comunicaciones ópticas inalámbricas, ciencias espaciales, internet de las cosas y el emprendimiento social.

ORCID: https://orcid.org/0000-0003-1634-066X

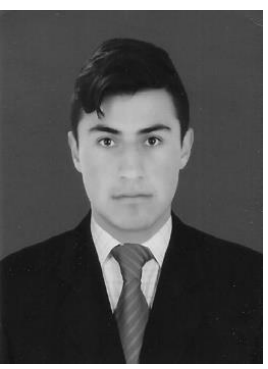

Germán Homero Morán Figueroa: Nació en Pupiales, Nariño, Colombia, el 15 de diciembre de 1996. Ingeniero en Electrónica y Telecomunicaciones de la Universidad del Cauca. Entre sus áreas de interés están sistemas de comunicaciones por fibra óptica, sistema de comunicaciones inalámbricos y seguridad en redes de información.

ORCID: https://orcid.org/0000-0002-3389-6750.

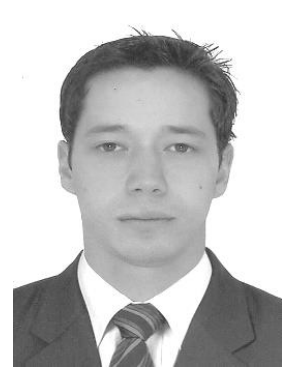

Gustavo Adolfo Gómez Agredo: Nació en Popayán, Cauca el 10 de junio de 1984. Ingeniero en Electrónica y Telecomunicaciones de la Universidad del Cauca, Especialista (c) en TIC para la Innovación Educativa de la Universidad del Cauca, Magister en Electrónica y Telecomunicaciones de la Universidad del Cauca, docente del departamento de telecomunicaciones, investigador del Grupo de Nuevas Tecnólogas en Telecomunicaciones - GNTT. Entre sus áreas de interés están: Comunicaciones por fibra óptica, Comunicaciones ópticas inalámbricas y

Radiocomunicaciones.

ORCID: https://orcid.org/0000-0002-3413-562X. 